\title{
Effective prevention bundle to eliminate catheter-related bloodstream infections in ambulatory hemodialysis patients
}

\author{
Hoda A. Hamid; ${ }^{1}$ Hicham Bouanane; ${ }^{1}$ Athar Ibrahim; ${ }^{1}$ Sahar Ismail; ${ }^{1}$ Aisha El Sayed; ${ }^{1}$ Khaled M. Mahmoud; ${ }^{2}$ Abdulla Hamad; ${ }^{2}$ \\ Fadwa Al Ali \\ ${ }^{1}$ Fahd Bin Jassim Kidney Center, Hamad General Hospital, Doha, Qatar \\ ${ }^{2}$ Nephrology Unit, Hamad General Hospital, Doha, Qatar
}

\section{Corresponding author:}

Khaled Mahmoud, MD, PhD, FASN

Nephrology Unit, Hamad General Hospital, PO 3050, Doha, Qatar

Tel.: 009744439599

kmohamed8@hamad.qa

\begin{abstract}
Background: Hamad General Hospital $(\mathrm{HGH})$ is the principal provider of dialysis in the state of Qatar, comprising a total of four facilities in different cities. Infection rates in dialysis patients are increasingly used as a surrogate marker for measuring patient safety and quality of healthcare. These infections are associated with substantial morbidity, mortality, and excess healthcare costs. We observed an elevated rate of hemodialysis catheter-related bloodstream infections (HD-CRBSI) in our outpatient dialysis facilities (1.4/1,000 Central Venous Catheter [CVC] days) in 2011. Our goal was to reduce our HD-CRBSI rate by $80 \%$ within a period of four years in HGH ambulatory dialysis facilities.
\end{abstract}

Methods: HD-CRBSIs are defined as the presence of positive blood cultures in a febrile catheter-dependent patient in the absence of alternative sources of infection upon clinical evaluation. The project was led by the HGH quality improvement program director in coordination with a multidisciplinary team (nephrologists, nurses, vascular coordinators, a patient educator, and an infection control team) after implementation of a bundle of infection prevention measures.

Results: The rate of HD-CRBSI was reduced from 1.4/1,000 CVC days in 2011 to 0.014 in 2017, achieving a 99\% reduction rate $(p<0.001)$.

Conclusions: Strict implementation of our new infection prevention measures bundle is sufficient to significantly reduce HD-CRBSIs.

\section{KEYWORDS}

Prevention; hemodialysis catheter; bloodstream; infection

\section{INTRODUCTION}

Minimization of line-related bloodstream infections and establishing dialysis access are often challenging tasks in hemodialysis facilities for patients requiring renal replacement therapy. A number of factors, including patient reluctance, anatomic host factors, and prolonged maturation time, have contributed to a preponderance of dialysis catheter use.

Many scientific societies and the U.S. Centers for Disease Control and Prevention have suggested different measures to prevent catheter-related bloodstream infections (CRBSIs) [1]. Many promising articles targeting the prevention of CRBSIs have been published since the publication of these guidelines in 2011.

One of the measures for prevention is to encourage fistula creation and usage. National quality improvement programs, which included the breakthrough Fistula First Initiative, have been shown to be ineffective and, in many instances, have contributed to many patients on hemodialysis (HD) initiating renal replacement therapy with a catheter.

Up to $80 \%$ of patients undergoing maintenance HD in the United States initiate treatment via a central venous catheter (CVC) with significantly more infections than arteriovenous fistulae or grafts [2].

CRBSIs were not well defined until 2009, when the Infectious Diseases Society of America recognized the unique characteristics of HD catheters. The definition relied on obtaining a blood specimen from the dialysis catheter and an additional specimen from a peripheral vein [3]. The existence of a similar colony count, differential, and time-to-sensitivity at both sites are the criteria for diagnosis of a CRBSI in the absence of alternative sources of infection upon clinical evaluation.

It should be noted that indwelling vascular catheters are colonized by microorganisms within 24 hours after their insertion. Bacteria are introduced into the lumen through 


\begin{tabular}{|c|c|c|}
\hline Current practice reviewed & New management practice developed & $\begin{array}{c}\text { New practice implemented and } \\
\text { evaluated }\end{array}$ \\
\hline $\begin{array}{l}\text { - Previous outcome studied } \\
\text { - Barriers identified: } \\
\text { - Patient level } \\
\text { - Staff level } \\
\text { - Management level } \\
\text { - Vascular access }\end{array}$ & $\begin{array}{l}\text { - Vascular access management quality } \\
\text { improvement project initiated } \\
\text { - Vascular access management protocol } \\
\text { developed } \\
\text { - Data collection tracking system created } \\
\text { - Vascular access educational care program } \\
\text { initiated }\end{array}$ & $\begin{array}{l}\text { The new practices were implemented } \\
\text { based on: } \\
\text { - Multidisciplinary team care evaluation } \\
\text { - Vascular access management protocol } \\
\text { reviewed and updated as needed } \\
\text { - Monitoring staff adherence to protocol } \\
\text { - Clinical practices measurements } \\
\text { - Tracking outcome data }\end{array}$ \\
\hline
\end{tabular}

the flora of the overlying skin or the hands of healthcare personnel during catheter manipulation via either connection or disconnection $[4,5]$.

In the state of Qatar, Hamad General Hospital $(\mathrm{HGH})$ is the principal provider of dialysis, comprising a total of four facilities located in different cities. In 2011, we observed an elevated rate of hemodialysis catheter-related bloodstream infections (HD-CRBSIs) in our outpatient dialysis facilities (1.4/1,000 CVC days).

\begin{abstract}
Aim
Infection rates in dialysis patients are increasingly used as a surrogate marker for measuring patient safety and quality of healthcare and are associated with substantial morbidity, mortality, and excess healthcare costs $[6,7]$. We targeted to reduce our HD-CRBSI rate by $80 \%$ within a period of five years in $\mathrm{HGH}$ ambulatory dialysis facilities.
\end{abstract}

\section{METHODS}

We planned to achieve our objective by applying a bundle of infection prevention measures. The project was implemented by the $\mathrm{HGH}$ quality improvement program director in coordination with a multidisciplinary team that included nephrologists, nurses, vascular coordinators, a patient educator, and an infection control team.

\section{Project design/strategy for change}

We started our project by reviewing our current practice, identifying instances where previous outcomes had been studied, and identifying barriers at all levels, including patient, staff, and management. Particular attention was given to reviewing vascular access management protocols.

While reviewing our current protocols, we also developed our new catheter management practice. Our vascular access quality improvement project, initiated in 2012, consisted of a new vascular access management protocol, a data collection system, a patient tracking system, and a vascular access educational care program.

Lastly, we conducted evaluation of the new practice, which consisted of multidisciplinary team care evaluation, assessment of the vascular access management protocol, monitoring of staff adherence to the new protocol, clinical practices measurements with real-time data, and outcome tracking.

\section{Changes made (the five tracks model)}

The following five tracks of changes have been implemented simultaneously:

1. Patient and family education efforts focused on general hand hygiene awareness and on early recognition of signs and symptoms of catheter infection. Patients and family members were advised to use a waterproof pocket to protect the catheter during swimming and showering. Educational materials were developed to improve their awareness of the importance of catheter access point care.

2. Nursing education: all of our dialysis facility nurses revalidated their competency for CVC care through mandatory infection control courses. Furthermore, change of catheter dressing was limited only to the qualified dialysis staff. As part of the project, dialysis staff initiated ongoing surveillance of catheter infection with the infectious disease team.

3. Reduction of permanent catheter insertion: reducing the number of permanent catheter insertions was achieved via adoption of the Fistula First program, with emphasis on maintaining patency of arteriovenous fistula (AVF)/arteriovenous graft by early intervention and close monitoring.

4. Management of catheter malfunction: we implemented the use of innovative catheter lock solutions such as taurolidine and recombinant tissue plasminogen activator locks.

5. Technique improvement: we implemented several changes to improve our techniques of access point management, including the introduction of chlorhexidine $2 \%$ to replace betadine and iodine on the catheter hub and exit site, respectively. We also introduced a chlorhexidine-impregnated Tegaderm ${ }^{\mathrm{TM}}$ dressing for seven-day use on the exit site. As well, a single sterile kit replaced a set of separate sterile items used at the start and termination of each dialysis session.

\section{RESULTS}

Our study included 99 patients dialyzed via permanent catheters, including 59 males and 40 females with a mean age of $55 \pm 14.8$ years. The study lasted for seven years, from 2011 to 2017 . Of the 99 patients, 89 patients had 
FIGURE 1: Hemodialysis catheter-related bloodstream infection rate in Hamad General Hospital dialysis units.

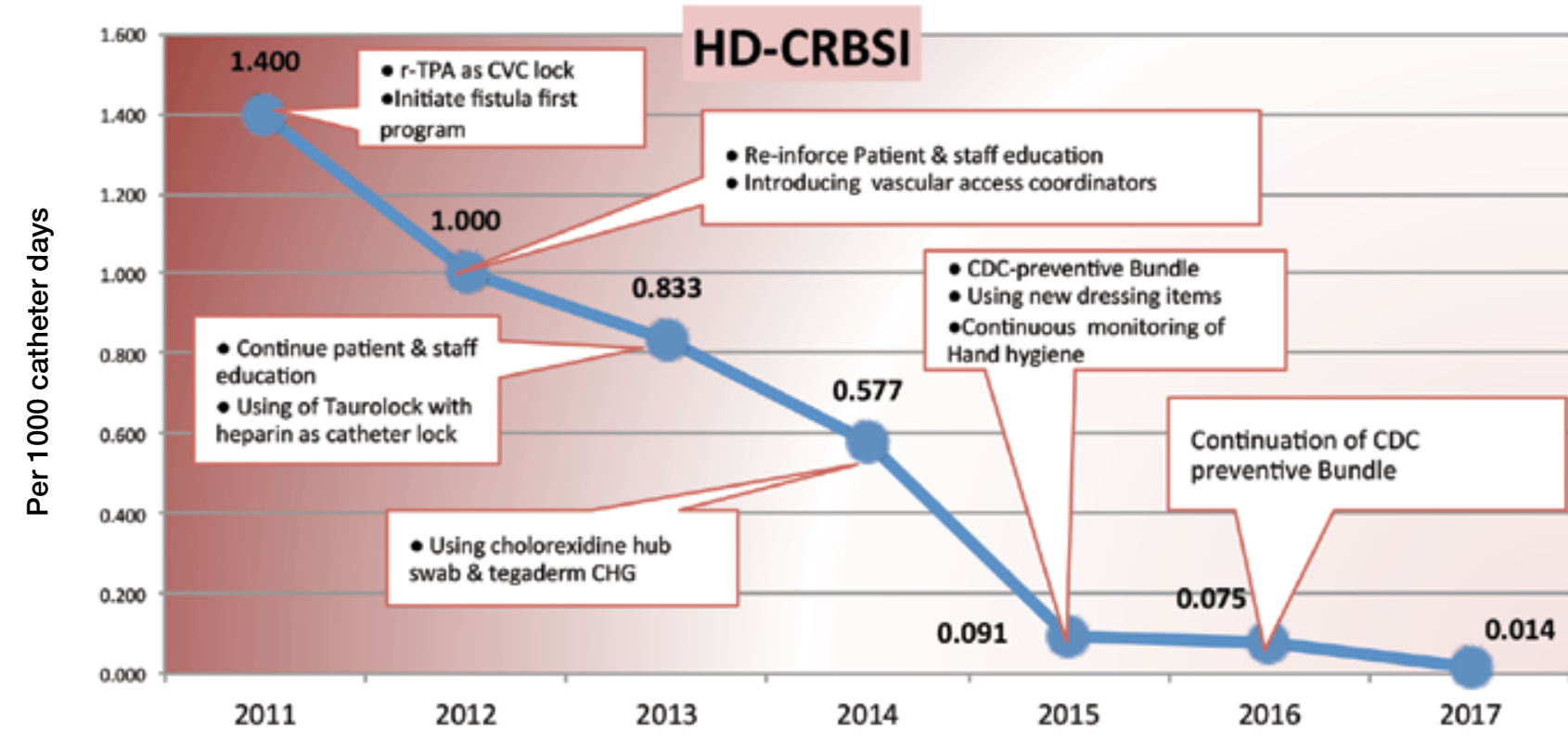

their catheters exchanged, six received antibiotic therapies and continued to use their catheters, two had their catheters removed and AVF created, and two more patients were lost to follow-up due to international travel. The most common organism found in our study population was Enterobacter cloacae, which constituted $15 \%$ of infections.

Figure 1 shows that after implementing the new infection prevention bundle, the rate of HD-CRBSI decreased from $1.4 / 1,000$ CVC days in 2011 to $0.014 / 1,000$ CVC days in 2017 , an overall reduction of $99 \%$.

\section{DISCUSSION}

CRBSIs are one of the most common bacterial infections in patients receiving hemodialysis treatments [8]. In our study, we successfully reduced the rate through an intervention bundle that proved to be effective against CRBSIs.

The success of the bundled intervention is largely attributable to a multidisciplinary team approach, which included participation from nephrologists, nurses, vascular coordinators, a patient educator, and infection control practitioners. Education of patients, families, and nursing staff has been previously reported to improve the outcome [9].

We have successfully overcome the problem of catheter malfunction with the use of new catheter lock solutions like taurolidine and recombinant tissue plasminogen activator locks. Olthof et al. (2014) appreciated the value of using taurolidine lock in their home parenteral nutrition group of patients, where it significantly reduced the rate of CRBSI and occlusion [10].

Our project has since transitioned into sustainability mode, placing greater emphasis on the evaluation of patient clinical outcomes such as hospitalization, mortality rates, and cost.

\section{REFERENCES}

1. Centers for Disease Control and Prevention. (2011). Vital signs: Central line-associated blood stream infections - United States, 2001, 2008, and 2009. Morbidity and Mortality Weekly Report, 60(8), 243248. Retrieved from https://www.ncbi.nlm.nih.gov/pubmed/21368740

2. U.S. Renal Data System. (2011). USRDS 2011 annual data report: Atlas of chronic kidney disease and end-stage renal disease in the United States. Retrieved from https://www.usrds.org/2011/view/default.asp.

3. O'Grady, N. P., Alexander, M., Burns, L. A., Dellinger, E. P., Garland, J., Heard, S. O., Lipsett, P. A., Masur, H., Mermel, L. A., Pearson, M. L., Raad, I. I., Randolph, A. G., Rupp, M. E., \& Saint, S. (2011). Summary of recommendations: Guidelines for the prevention of intravascular catheter-related infections. Clinical Infectious Diseases, 52(9), 10871099. doi: $10.1093 / \mathrm{cid} / \mathrm{cir} 138$

4. Raad, I., Costerton, W., Sabharwal, U., Sacilowski, M., Anaissie, E., \& Bodey, G. P. (1993). Ultrastructural analysis of indwelling vascular catheters: A quantitative relationship between luminal colonization and duration of placement. Journal of Infectious Diseases, 168(2), 400-407. Retrieved from https://www.ncbi.nlm.nih.gov/ pubmed/8335977

5. Marr, K. A., Sexton, D. J., Conlon, P. J., Corey, G. R., Schwab, S. J., \& Kirkland, K. B. (1997). Catheter-related bacteremia and outcome of attempted catheter salvage in patients undergoing hemodialysis. Annals of Internal Medicine, 127(4), 275-280. Retrieved from https:// www.ncbi.nlm.nih.gov/pubmed/9265426

6. Gahlot, R., Nigam, C., Kumar, V., Yadav, G., \& Anupurba, S. (2014) Catheter-related bloodstream infections. International Journal of Critical Illness \& Injury Science, 4(2), 162-167. doi: 10.4103/22295151.134184

7. Soi, V., Moore, C., Kumbar, L., \& Yee, J. (2016). Prevention of catheter-related bloodstream infections in patients on hemodialysis: Challenges and management strategies. International Journal of Nephrology and Renovascular Disease, 9, 95-103. doi: https://doi. org/10.2147/IJNRD.S76826

8. Centers for Medicare and Medicaid Services. (2007). 2007 annual report: ESRD clinical performance measures project. Retrieved from https://www.cms.gov/Medicare/End-Stage-Renal-Disease/CPMProject/ downloads/ESRDCPMYear2007Report.pdf 
9. McAlearney, A. S., \& Hefner, J. L. (2014). Facilitating central lineassociated bloodstream infection prevention: A qualitative study comparing perspectives of infection control professionals and frontline staff. American Journal of Infection Control, 42(10 Suppl), S216-22. doi: 10.1016/j.ajic.2014.04.006

10. Olthof, E. D., Versleijen, M. W., Huisman-de Waal, G., Feuth, T., Kievit, W., \& Wanten, G. J. (2014). Taurolidine lock is superior to heparin lock in the prevention of catheter related bloodstream infections and occlusions. PLoS One, 9(11), e111216. doi: 10.1371/journal. pone.0111216 